\title{
Frequência de aleitamento materno exclusivo e razões para liberação de fórmula láctea em alojamento conjunto de uma maternidade de nível terciário
}

\author{
Frequency of exclusive breastfeeding and reasons for release of milk formula in \\ rooming-in of a tertiary-level maternity hospital
}

\author{
Fabíola Roberta Marim Bianchini ${ }^{1}$, Valdenise Martins Laurindo Tuma Calil' \\ Laura Matucci Tardelli ${ }^{3}$, Rebeca Lopes Dias ${ }^{3}$, Rossana Pulcineli Vieira Francisco ${ }^{4}$, \\ Werther Brunow de Carvalho ${ }^{5}$, Vera Lúcia Jornada Krebs ${ }^{6}$
}

\begin{abstract}
Bianchini FRM, Calil VMLT, Tardelli LM, Dias RL, Francisco RPV, Carvalho WB, Krebs VLJ. Frequência de aleitamento materno exclusivo e razões para liberação de fórmula láctea em alojamento conjunto de uma maternidade de nível terciário / Frequency of exclusive breastfeeding and reasons for release of milk formula in rooming-in of a tertiary-level maternity hospital. Rev Med (São Paulo). 2017 out.-dez.;96(4):215-9.

RESUMO: Introdução: O leite materno é fonte incomparável de nutrientes nos primeiros seis meses de vida e, após este período, recomenda-se que seja complementado com outros alimentos até dois anos ou mais. Publicações recentes evidenciam um aumento da prevalência de aleitamento materno (AM) exclusivo nos binômios assistidos em serviços onde se pratica o atendimento humanizado, como o sistema de alojamento conjunto (AC). Objetivo: Descrever a prevalência de $\mathrm{AM}$ e as razões consideradas para liberação de fórmula láctea para recém-nascidos de termo (FLT) no alojamento conjunto de uma instituição hospitalar pública de nível terciário. Casuística e métodos: Foi realizado um estudo retrospectivo descritivo em recém-nascidos encaminhados ao AC do Hospital das Clínicas da Faculdade de Medicina da Universidade São Paulo, instituição de nível terciário de atendimento, no período de junho a dezembro de

2016. Os dados foram obtidos através de revisão de prontuários e do protocolo instituído para coleta de dados. A internação no $\mathrm{AC}$ e as justificativas para liberação de FLT seguiram as respectivas normas institucionais. Resultados: No período estudado foram internados 555 recém-nascidos. A frequência de AM exclusivo foi de 70,8\%; a FLT foi liberada, portanto, em 29,2\% dos recém-nascidos. A principal razão para a indicação de FLT foi a hipoglicemia, correspondendo a $54,4 \%$ do total de FLT prescrito. As patologias maternas ocuparam o segundo lugar entre as justificativas mais frequentes, sendo citadas em $18,5 \%$ dos casos. Conclusão: As principais causas para prescrição de FLT foram hipoglicemia e patologias maternas. Estas expressam as características da população estudada, composta por filhos de mães com doenças graves que potencialmente impedem a amamentação ou favorecem condições clínicas hipoglicemiantes. O sistema de AC
\end{abstract}

1. Centro Neonatal, Instituto da Crianca, Hospital das Clinicas HCFMUSP, Faculdade de Medicina FMUSP, Universidade de Sao Paulo, Sao Paulo, SP, BR. Médica Pediatra e Neonatologista. Médica Assistente do Centro Neonatal do Instituto da Criança do HCFMUSP.

2. Centro Neonatal, Instituto da Crianca, Hospital das Clinicas HCFMUSP, Faculdade de Medicina FMUSP, Universidade de Sao Paulo, Sao Paulo, SP, BR. Médica Pediatra e Neonatologista. Doutora em Pediatria. Médica Assistente do Centro Neonatal do Instituto da Criança do HCFMUSP. Email: valdenise.calil@hc.fm.usp.br.

3. Faculdade de Medicina FMUSP, Universidade de Sao Paulo, Sao Paulo, SP, BR. Graduanda do curso de Medicina da FMUSP. Email: tardellilaura@gmail.com, rebecald3@hotmail.com.

4. Departamento de Obstetrícia e Ginecologia, Faculdade de Medicina FMUSP, Universidade Sao Paulo. Professora livre Docente da Disciplina de Obstetrícia. Email: rossana.francisco@hc.fm.usp.br.

5. Instituto da Crianca, Hospital das Clinicas HCFMUSP, Faculdade de Medicina FMUSP, Universidade de Sa Paulo, São Paulo, SP, BR. Professor Titular de Terapia Intensiva - Neonatologia do Instituto da Criança - HCFMUSP. Email: werther.brunow@hc.fm.usp.br.

6. Centro Neonatal, Instituto da Crianca, Hospital das Clinicas HCFMUSP, Faculdade de Medicina FMUSP, Universidade de Sao Paulo, Sao Paulo, SP, BR. Professora Livre Docente de Pediatria da FMUSP. Médica Chefe do Centro Neonatal do Instituto da Criança do HCFMUSP. Email: vera.krebs@hc.fm.usp.br.

Endereço para correspondência: Fabíola Roberta Marim Bianchini. Av. Dr. Enéas de Carvalho Aguiar, 255, 10 andar. CEP: $05403-000$ Cerqueira Cesar, São Paulo, SP, BR. Email: fabiola.bianchini@hc.fm.usp.br. 
e a notificação sistemática da justificativa para a liberação de FLT contribuíram para garantir que a mesma ocorresse apenas quando necessária, favorecendo a manutenção do AM .

Descritores: Alojamento conjunto; Recém-nascido; Aleitamento materno; Leite humano. Substitutos do leite humano.

ABSTRACT: Introduction: Breast milk is an unrivaled source of nutrients in the first six months of life, and after this time it is recommended that it must be supplemented with other foods up to two years or longer. Recent publications show an increase in the prevalence of exclusive breastfeeding in binomials that have been assisted in services in which humanized care is practiced, such as rooming-in (RI) system. Objectives: To describe the prevalence of breastfeeding (BF) and the reasons for the prescription of milk formula (MF) in the RI from a tertiary-level public hospital institution. Patients and methods: A descriptive retrospective study was carried out at the Hospital das Clinicas, Faculdade de Medicine, Universidade de Sao Paulo, a tertiary level of care institution, from June to

\section{INTRODUÇÃO}

$\mathrm{O}$ leite materno é fonte incomparável de nutrientes nos primeiros seis meses de vida e após este período, recomenda-se que seja complementado com outros alimentos até dois anos ou mais. Favorece assim, a construção de um futuro saudável para estes recém-nascidos, promovendo menor prevalência de diabetes melito, hipercolesterolemia, doença cardíaca isquêmica, hipertensão arterial crônica, doenças alérgicas e autoimunes, doença pulmonar obstrutiva crônica, obesidade e alguns tipos de linfomas e leucemias ${ }^{1}$.

Existem ainda evidências de que o aleitamento materno (AM), desde os primeiros meses de vida, resulta em maior quociente de inteligência a longo prazo ${ }^{2}$.

Publicações recentes evidenciam um aumento da prevalência de aleitamento materno exclusivo (AME) em binômios que foram assistidos em serviços onde se pratica o atendimento humanizado, como o alojamento conjunto $(\mathrm{AC})^{3}$.

O primeiro relato AC ocorreu em 1946, tendo o projeto sido idealizado por Edith Jackson, no Grace New Haven Hospital. Esse experimento mantinha mãe e recém-nascido (RN) juntos após o parto e surgiu como uma demanda da época de preparar as mães para cuidar de seus filhos. A iniciativa foi um sucesso e difundiu-se rapidamente para outros países. No Brasil, a primeira experiência de AC ocorreu em 1971, no Primeiro Hospital Distrital de Brasília, tornando-se recomendação do Ministério da Saúde apenas em 1977. Desde então, sua importância para manutenção do AM foi consagrada e adotada por várias unidades neonatais do país ${ }^{4}$.

$\mathrm{O} \mathrm{AC}$ favorece a precocidade, intensidade e assiduidade do AM. Seu caráter educativo permite corrigir falhas relacionadas ao manejo da lactação e com isso, garantir que a liberação de fórmulas lácteas (FLT) ocorra apenas quando houver indicação médica ${ }^{3}$.
December 2016. Data were obtained through a review of charts and the protocol established for data collection. The hospitalization in the $\mathrm{RI}$ and the justifications for the release of MF followed the respective institutional norms. Results: During the study period, 555 newborns were hospitalized. The frequency of exclusive breastfeeding was $70.8 \%$; the MF was therefore prescribed in $29.2 \%$ of newborns. The main reason described for the indication of MF was hypoglycaemia, corresponding to $54.4 \%$ of the total of offered MF. Maternal diseases ranked second among the most frequent justifications, being cited in $19 \%$ of the cases. Conclusion: The main causes for MF prescription were hypoglycaemia and maternal diseases. They express the features of studied population, represented by children of mothers with serious diseases that potentially prevent breastfeeding or favor hypoglycemic clinical conditions. The rooming-in system and the systematic notification of the reasons for use of MF contributed to ensure that it occurred only when necessary, favoring maintenance of the BF.

Keywords: Rooming-in care; Infant, newborn; Breast feeding; Milk, human; Breast-milk substitutes.

$\mathrm{O}$ artigo pretende descrever a prevalência de AME e as razões para liberação de fórmula láctea no AC de uma maternidade de nível terciário de atendimento.

\section{CASUÍSTICA E MÉTODOS}

Estudo retrospectivo descritivo realizado no AC do Hospital das Clínicas da Faculdade de Medicina da Universidade São Paulo, instituição classificada como nível terciário de atendimento, no período de junho de 2016 a dezembro de 2016. Foi estabelecido um protocolo para acompanhamento da utilização de FLT em todos os recém-nascidos internados no AC. Os dados foram obtidos através de revisão de prontuários e do protocolo instituído.

As gestantes internadas na unidade possuíam em sua maioria, elevado risco obstétrico. Dentre as patologias observadas nas puérperas admitidas no AC pode-se citar: hipertensão arterial sistêmica, câncer de mama e outras doenças oncológicas, insuficiência hepática ou renal com necessidade de transplante, trombocitopenias, colagenoses, coagulopatias, cardiopatias congênitas e reumáticas, diabetes gestacional e melito, esclerose múltipla, esclerose tuberosa, psicopatologias e epidermólise bolhosa. Os recém-nascidos apresentavam repercussões clínicas decorrentes das condições maternas descritas, tais como restrição de crescimento, prematuridade, asfixia, distúrbios metabólicos, icterícia, entre outras.

A indicação de encaminhamento do RN para o AC foi realizada a partir da portaria $\mathrm{n}^{\circ} 2.068$ de 21 de outubro de 2016, adaptada às características do binômio mãe/filho da instituição ${ }^{5}$.

Foram elegíveis para o AC mães e recém-nascidos com o seguinte perfil:

- Mães clinicamente estáveis e sem contraindicações para permanência junto ao RN;

- Recém-nascidos com boa vitalidade, capacidade de sucção e controle térmico; 
- Recém-nascidos com peso de nascimento igual ou superior a 2.200 gramas;

- Recém-nascidos com idade gestacional igual ou superior a 35 semanas;

- Recém-nascidos com índice de APGAR maior que 6 no $5^{\circ}$ minuto;

- Recém-nascidos livres de condições clínicas que necessitem de cuidados especiais (oxigênio suplementar, uso de antibióticos ou de soro endovenoso e outras);

- Recém-nascidos com ausência de doenças hemolíticas;

- Recém-nascidos sem malformações maiores.

A FLT foi liberada após prescrição médica, que seguiu o protocolo da instituição descrito abaixo, adaptado do documento "Razões médicas aceitáveis para o uso de substitutos do leite materno, OMS, 2009"6:

\section{- Condições da criança}

1) Recém-nascidos que não devem receber leite materno nem qualquer outro leite, exceto fórmulas especiais:

a) Galactosemia clássica: fórmula especial isenta de galactose;

b) Doença da urina de xarope do bordo: fórmula especial livre de leucina, isoleucina ou valina;

c) Fenilcetonúria: fórmula especial isenta de fenilalanina.

2) Recém-nascidos para os quais o leite materno é a melhor opção de alimento, mas que podem necessitar de complementação com outro leite por período limitado:

a) Controle de glicemia pré-mamada (fita reagenteDextrostix $) \leq 45 \mathrm{mg} / \mathrm{dl}^{8,9}$, valor este mantido após duas tentativas de recuperação glicêmica através do AM;

- Hipoglicemia em virtude de adaptação metabólica comprometida ou demanda aumentada de glicose (RN pré-termo, pequeno para a idade gestacional, grande para a idade gestacional, filho de mãe diabética, asfixiado grave).

b) Sinais clínicos de desidratação:

- Febre

- Ausência de diurese por período maior que $12 \mathrm{~h}$ (exceção do $1^{\circ}$ dia de vida).

c) Perda de peso superior a $10 \%$ do peso de nascimento nas primeiras $48 \mathrm{~h}$ de vida.

\section{- Condições maternas}

1) As mães afetadas por qualquer das condições abaixo devem receber tratamento de acordo com procedimento padrão:

a) Condições que contra-indicam o AM de forma permanente (HIV, HTLV);

b) Condições que contra-indicam o AM de forma temporária (sepse; vírus do Herpes Simples tipo 1 ; Varicela);

c) Medicações (sedativos, psicotrópicos, antiepiléticos, opiáceos e suas combinações);

d) Radioterapia;

e) Uso em excesso de iodo ou iodo for tópico;

f) Quimioterapia citotóxica.

2) Condições maternas durante as quais amamentar não é contra-indicado, embora representem problemas de saúde que causam preocupação ou impossibilidade de manter o AME:

a) Abscesso mamário;

b) Mastite;

c) Mamoplastia; mastectomia;

d) Hepatite B: os lactentes devem receber vacina contra a Hepatite B nas primeiras $48 \mathrm{~h}$ de vida;

e) Hepatite C;

f) Tuberculose (mãe e RN devem ser tratados em conjunto);

g) Uso de certas substâncias: nicotina, álcool, ecstazy, anfetaminas, cocaína e estimulantes relacionados, benzodiazepínicos.

O documento "Razões médicas aceitáveis para o uso de substitutos do leite materno, OMS, 2009"6 serviu de base para o protocolo de liberação de FLT do serviço, tento sofrido algumas adaptações para se adequar às particularidades da população estudada. São elas:

- Relacionadas às condições da criança: sinais clínicos de desidratação e perda de peso superior a $10 \%$ nas primeiras 48 horas de vida;

- Relacionadas às condições maternas: mamoplastia e mastectomia.

Todos os recém-nascidos que receberam FLT, mesmo que ofertada uma única vez, foram incluídos no estudo de acordo com as justificativas contidas no prontuário. A frequência de utilização de FLT foi apresentada em porcentagens do total de internações no AC.

\section{RESULTADOS}

No período estudado foram encaminhados 555 recém-nascidos ao $\mathrm{AC}$, selecionados de acordo com o protocolo descrito acima.

A frequência de AME foi de 70,8\% (n=393); a FLT foi liberada, portanto, em 29,2\% $(n=162)$ dos recémnascidos (Gráfico 1).

A principal razão citada para a indicação de FLT foi a hipoglicemia $(n=88)$, correspondendo a $54,4 \%$ do total de FLT prescrito. As patologias maternas ocuparam o segundo lugar dentre as justificativas mais frequentes $(n=30)$, sendo citadas em 18,5\% dos casos (Gráfico 2). 
Gráfico 1. Frequência de aleitamento materno e fórmula láctea de termo no alojamento conjunto

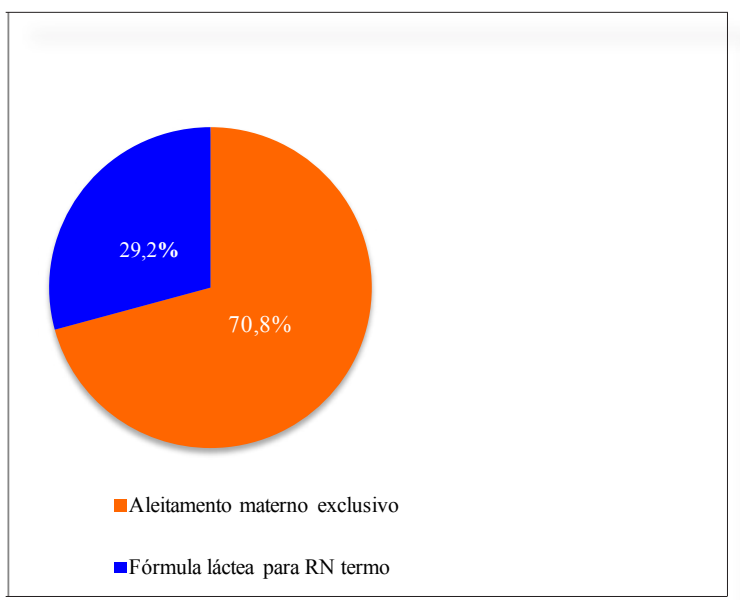

As demais justificativas encontradas e suas respectivas porcentagens estão descritas no Gráfico 2.

\section{DISCUSSÃO}

O AC é um sistema hospitalar no qual o RN, de preferência imediatamente após o nascimento, permanece ao lado da mãe 24 horas por dia num mesmo ambiente até a alta hospitalar. Durante a internação o binômio é atendido por uma equipe multidisciplinar que fortalece as orientações das mamadas ao seio materno e corrige erros que possam surgir com o retorno das famílias às suas residências ${ }^{3}$. O método esta incluído no passo 7 dos Dez Passos para o Sucesso do Aleitamento Materno, inseridos nas condições para a implantação da Iniciativa Hospital Amigo da Criança (IHAC) ${ }^{7}$.

Gráfico 2 - Justificativas para liberação de fórmula láctea termo no alojamento conjunto

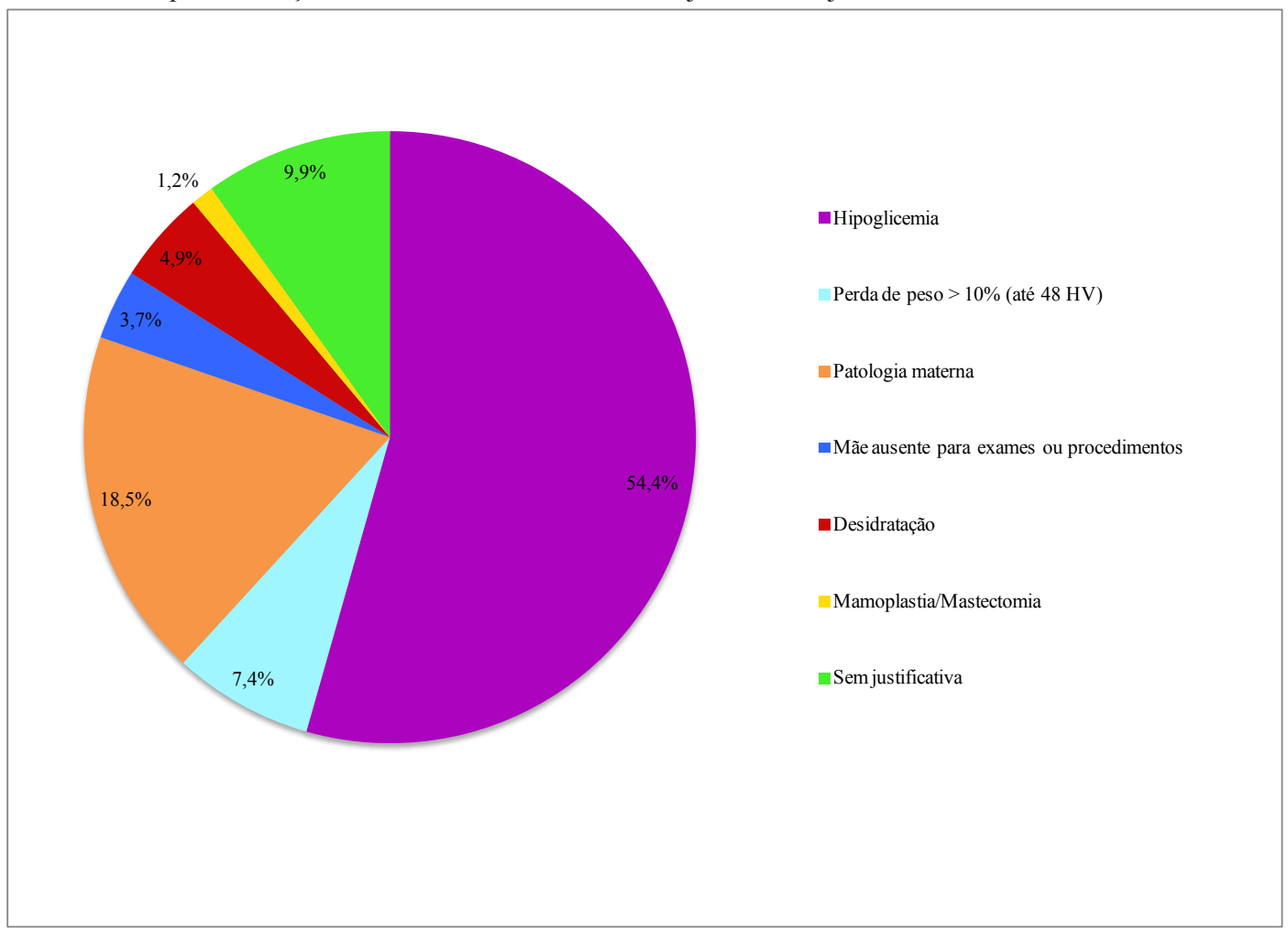

A IHAC tem como objetivo principal favorecer o AM e reduzir os altos índices de desmame precoce. Para atender aos pré-requisitos da iniciativa são desejáveis taxas de AME iguais ou superiores a 75\% durante a internação e a liberação de FLT somente por razões médicas aceitas pela OMS. A utilização do sistema de AC neste serviço, classificado como nível terciário de atendimento por prestar assistência a puérperas com elevado risco obstétrico, possibilitou a frequência de $70,8 \%$ de AME até a alta hospitalar.

A análise dos prontuários permitiu observar que apenas $9,9 \%$ dos casos de liberação da FLT não respeitaram as justificativas incluídas na norma da instituição; destes, $7 \mathrm{RN}$ (4,3\%) eram gemelares. Os pares de gemelares podem ser encaminhados para o AC desde que respeitem os critérios de peso e idade gestacional estabelecidos no protocolo do serviço; no entanto, a manutenção de ambos em AME, embora possível, não é tarefa fácil em quaisquer condições, especialmente em puérperas que tiveram gestações de alto risco. Portanto, a taxa restante de FLT não justificadas $(5,6 \%)$ representou, em nossa opinião, um resultado positivo.

Dentre as principais causas de liberação de FLT destacaram-se a hipoglicemia e as patologias maternas, 
achado concordante com as características da população de pacientes internada na instituição.

No que se refere à hipoglicemia $(54,4 \%)$, o valor de corte para sua definição $(\leq 45 \mathrm{mg} \%)$ foi baseado nas recomendações do Committee on Fetus and Newborn Infants Clinical ${ }^{9}$, e nas recentes diretrizes da Sociedade Brasileira de Pediatria ${ }^{8}$. Estes níveis são considerados bastante seguros para RN em geral e particularmente para aqueles nas condições clínicas descritas. O controle glicêmico por fita reagente (Dextrostixr ${ }^{\circledR}$ ) foi indicado para recém-nascidos com restrição de crescimento intrauterino, filhos de mães diabéticas, grandes para a idade gestacional e asfixiados; a prescrição da FLT só ocorreu após tentativa mal sucedida de normalização da glicemia com AM.

Foi observado ainda que, dentre as justificativas citadas para prescrição de FLT, houve 4,9\% de "sinais clínicos de desidratação" e 7,4\% de "perda de peso superior a $10 \%$ nas primeiras 48 horas de vida". Estes itens não estão contidos no documento original do Ministério da Saúde ${ }^{6}$, mas a frequência com que foram descritos revelou sua importância nos recém-nascidos estudados.

O composto lácteo foi autorizado, portanto, com muito critério e seguindo rigorosamente o protocolo da instituição.

\section{REFERENCIAS}

1. Chaves RG. Por que amamentar exclusivamente até 6 meses e manter a amamentação até 2 anos ou mais?. In: Santiago LB, editor. Manual de aleitamento materno DCAM - SBP. Barueri: Manole; 2013. p.21-30.

2. Victora CG, Horta BL, Mola CL de, Quevedo L, Pinheiro RT, Gigante DP. Association between breastfeeding and intelligence, educational attainment, and income at 30 years of age: a prospective birth cohort study from Brazil. Lancet. 2015;3(4):199-205. doi: 10.1016/S2214-109X(15)70002-1.

3. Brasil. Ministério da Saúde. Secretaria de Atenção à Saúde. Departamento de Ações Programáticas Estratégicas. Cuidados no alojamento conjunto. In: Brasil. Ministério da Saúde. Atenção à saúde do recém-nascido: guia para os profissionais de saúde. 2a ed. atual. Brasília, DF; 2014. v.3, p.83-95. Disponível em: http://bvsms.saude.gov.br/bvs/publicacoes/ atencao_saude_recem_nascido_v3.pdf.

4. Ungerer RLS, Miranda ATC. História do alojamento conjunto. J Pediatr (Rio J.). 1999;75(1):5-10. Disponível em: http:// www.jped.com.br/conteudo/99-75-01-05/port.pdf.

5. Brasil. Ministério da Saúde. Portaria n ${ }^{\circ} 2.068$, de 21/10/2016 sobre diretrizes para a organização da atenção integral e

\section{CONCLUSÃO}

As justificativas citadas com maior frequência pelos médicos em prontuário, hipoglicemia e patologias maternas, concordaram com as "razões médicas aceitáveis para uso de substitutos do leite materno (OMS)" ". Elas expressaram as características da nossa população, representadas por filhos de mães com patologias graves que potencialmente impediram a amamentação ou que favoreceram condições clinicas hipoglicemiantes (restrição de crescimento, prematuridade tardia, asfixia e hiperinsulinismo).

Outras causas consideradas relevantes para liberação de FLT foram "sinais clínicos de desidratação" e "perda de peso superior a $10 \%$ nas primeiras 48 horas de vida", itens não contidos no documento original do Ministério da Saúde .

O sistema hospitalar de Alojamento Conjunto e a notificação sistemática da justificativa para a liberação de FLT contribuíram para garantir que a mesma ocorresse apenas quando necessária, favorecendo a manutenção do AM.

humanizada à mulher e ao recém-nascido no alojamento conjunto. Diário Oficial da União, 2016 out 24; n 204 , seção 1, p.121. Disponível em: http://www.poderesaude.com.br/ novosite/images/24.10.2016_I.pdf.

6. Organização Mundial da Saúde (OMS). Razões médicas aceitáveis para uso de substitutos do leite materno. Atualização OMS, 2009. Disponível em: http://apps.who.int/ iris/bitstream/10665/69938/2/WHO_FCH_CAH_09.01_por. pdf.

7. Brasil. Ministério da Saúde. Portaria $n^{0} 1.153$ de 22/05/2014 sobre redefinição dos critérios de habilitação da Iniciativa Hospital Amigo da Criança (IHAC). Disponível em: http://bvsms.saude.gov.br/bvs/saudelegis/gm/2014/ prt1153_22_05_2014.html.

8. Sociedade Brasileira de Pediatria. Departamento Científico de Endocrinologia. Diretrizes SBP - Hipoglicemia no período neonatal. SBP; 2016. Disponível em: http://www.sbp. com.br/fileadmin/user_upload/2016/09/Hipoglicemia-DCEndocrino-OK.pdf.

9. Adamkin DH, Committee on Fetus and Newborn infants Clinical. Postnatal glucose homeostasis in late-preterm and term. Pediatrics. 2011;127(3):575-9. doi:10.1542/peds.20103851 . 